\title{
Nickel zinc ferrite thick film for optimized performance of flexible patch antenna
}

\begin{abstract}
This work investigated the effect of adding nickel zinc ferrite thick film layer to a flexible substrate as overlay below a conductive silver patch. The ferrite thick film paste was prepared by mixing nickel zinc ferrite (NZF) nanopowders with an organic vehicle, and later using screen printing technique to print a thick film layer onto polyimide film, and later added a rectangle silver conductive patch on top of the ferrite layer. Measurement results of the flexible patch antenna with NZF thick film layer show that the thick film significantly widens the bandwidth of the antenna while having good return loss. The bending test, however, showed that there are significant changes of frequency and return loss when the antenna was bent at different angles, showing that further improvement is needed to further stabilizes the antenna parameters in bent conditions.
\end{abstract}

Keyword: Thick film; Flexible antenna; Nickel zinc ferrites; Ferrites 